\section{O Campo Transnacional da Inovação Tecnológica e Estratégias Relacionais de Ação}

\author{
Paulo Cesar Matui, Roniberto Morato do Amaral, Mário Sacomano Neto \\ e Silvio Eduardo Alvarez Candido
}

\section{RESUMO}

O objetivo deste artigo é analisar a influência de novas tecnologias na estrutura relacional do campo da indústria automotiva. Estamos usando uma construção dos componentes fortemente conectados (Strong Connected Component- SCC) como proxy de um campo de ação. Trata-se de uma simulação fundamentada nos dados extraídos de 7.211 registros de patentes tetraédricas - patentes com prioridade registrada simultaneamente na China, EUA, Europa e Japão. Adotamos uma forma descritiva da ação estratégica num campo modelado como uma rede. A partir desta rede apresentamos os dados em painéis e buscamos identificar alterações estruturais dos grupos e da métrica nodal da intermediação. Também realizamos análise semântica para simular o padrão de escolhas dos atores acerca de concepções tecnológicas concorrentes e apresentamos os dados em painéis que identificam o padrão de escolha tecnológica antes e depois da entrada do Google. As estruturas relacionais analisadas nesta pesquisa nos mostraram que a formação de grupos hierárquicos em SCC evidencia a ação estratégica das montadoras em estabilizar o campo da inovação tecnológica. Encontramos evidências de ação relacional como forma de enquadrar a ação de concorrentes e suas escolhas. Identificamos uma participação marginal dos atores da Tecnologia da Informação e Comunicações (TIC), e que a inovação desse campo é sobretudo arquitetural.

Palavras-Chave: redes; campos, sistemas de inovação, carro autônomo; tecnologia da informação

\section{The Transnational Field of Technology Innovation and The Relational Strategic Actions}

\section{ABSTRACT}

The purpose of this article is to analyze the influence of new technologies on the relational structure of the automotive field. We are using a Strong Connected Component (SCC) as a proxy for an action field. This is a simulation based on the data extracted from 7,211 tetrahedral patents - which is simultaneously valid with China, US, Europe and Japan. We have adopted a descriptive form of strategic action in a field modeled as a network. From this network we present the data in panels to identify the structural changes of the groups and the nodal metric of the betweenness. We also perform semantic analysis to simulate the pattern of the actors' choices about technological concepts and present the data in panels that identify the pattern of technological choices before and after Google's entry. The relational structures analyzed in this research showed that the formation of hierarchical groups in SCC shows the strategic action of automakers in stabilizing the field of technological innovation. We found evidence of relational action as a way of framing the action of competitors and their choices. We identified a marginal participation of the actors of Information Technology and Communications (ICT), and that the innovation of this field is mainly architectural.

Keywords: networks; fields; innovation systems; autonomous car; information technology

Recebido em: 07/05/2018 Revisado em: 31/05/2018 Aprovado em: 05/11/2018

Check for updates

\begin{abstract}
Paulo Cesar Matui iD, Programa de Pós-Graduação em Engenharia de Produção, Universidade Federal de São Carlos, Brasil Doutor em Engenharia de Produção, Universidade Federal de São Carlos, Brasil
\end{abstract}

paulo.matui@gmail.com

Roniberto Morato do Amaral (iD,

Programa de Pós-Graduação em Ciência da Informação, Universidade Federal de São Carlos, Brasil Doutor em Engenharia de Produção, Universidade Federal de São Carlos, Brasil

roniberto@ufscar.br

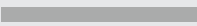

Mário Sacomano Neto (iD, Programa de Pós-Graduação em Engenharia de Produção, Universidade Federal de São Carlos, Brasil Doutor em Engenharia de Produção, Universidade Federal de São Carlos, Brasil

msacomano@ufscar.br

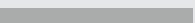

Silvio Eduardo Alvarez Candido (D),

Programa de Pós-Graduação em Engenharia de Produção, Universidade Federal de São Carlos, Brasil Doutor em Engenharia de Produção, Universidade Federal de São Carlos, Brasil

seacandido@dep.ufscar.br 


\section{Introdução}

Ao se falar em desenvolvimento tecnológico, como a transformação que está em curso na indústria automotiva com veículos autônomos, direção assistida, baliza assistida, veículos híbridos e veículos elétricos por exemplo, muitas vezes é usado o adjetivo disruptivo para descrever tais transformações. Porém, o que temos vivenciado são transformações não disruptivas. Novas tecnologias que poderiam mudar tudo, mas na prática não mudam nada, ou quase nada na estrutura socioeconômica do setor. Quase nada porque no âmbito do trabalho a história promete ser diferente. Todavia, neste estudo não é nosso objetivo abordar o tema do trabalho, uma vez que nosso tema aqui é a inovação. Vamos nos concentrar em entender a intersecção de dois setores industriais, a indústria automotiva e a tecnologia da informação e comunicações - a partir de agora referenciada como TIC.

Também é necessário entender que o nível organizacional é apenas parte do jogo. As ações que as organizações realizam individualmente fazem parte do processo, assim como o que o estado regula e incentiva também faz, mas o que tem sido representativo é como os atores desse espaço enquadram as ações uns dos outros. Enfim, trata-se de um jogo disputado num espaço chamado campo (Fligstein \& McAdam, 2012), e neste nível vamos encontrar uma competição amarrada por uma complexa rede (Lazzarini, 2011) composta sobretudo por relações de poder e de autopreservação. Vale ressaltar que, muitas vezes, não vemos o que realmente está alavancando as mudanças.

Um conceito que vem se mostrando relevante para explicar as mudanças sociais que temos presenciado é o conceito de sistemas de inovação tecnológica (technological innovation systems - TIS). Tal conceito começou a ser cunhado a partir da década de 1980 (Lundvall, 2005), e propõe a interligação de setores governamentais, científicos e industriais nacionais e transnacionais como forma de explicar sistemicamente a inovação, a qual se compõe num processo que ocorre necessariamente na interação de setores (Binz \& Truffera, 2017; Schuelke-Leech, 2018). Dentre seus estudos, um exemplo importante é o transbordo ou contágio tecnológico do setor químico para telecomunicações, e automotivo com o desenvolvimento de baterias que atendam necessidades de alta densidade de energia armazenada (Dijk, PeterWells, \& Kemp, 2016). Isso também nos mostra que é correto pensar em uma plataforma inovadora que não será uma concepção pura de nenhum setor específico, mas uma construção de atores socialmente e tecnologicamente qualificados (Hölzl \& Janger, 2014). Além disso, a conexidade, mais precisamente a proximidade, afeta o desempenho organizacional num arranjo denominado quase-campo de poder (Lee, Bachrach, \& Lewis, 2014). Vale ressaltar que há o estudo que analisou um arranjo a partir de redes semânticas para entender como o campo discursivo exerce influência simbólica sobre a inovação organizacional (Bail, 2014). Portanto, a inovação vai ocorrer num arranjo relacional entre os setores governamentais, científicos e industriais. 
Neste estudo aproximaremos a teoria de campos de ação estratégica e o TIS (Franssen \& Kuipers, 2013; Mitnick \& Ryan, 2015; Fellows \& Liu, 2016; Marti, 2017). A medida que as empresas se aproximam da fronteira de conhecimento tecnológico, elas precisam cada vez mais se concentrar na criação de conhecimento próprio e na adoção de estratégias de crescimento baseadas na inovação para se manterem competitivas (Hölzl \& Janger, 2014). Em nossa perspectiva isso ocorre com o estabelecimento de um campo de ação cujo principal ativo é o domínio da tecnologia. O que nos leva a crer que se trata de um projeto cultural, expresso nas escolhas tecnológicas e em sua propriedade intelectual, na forma relacional de governança e na forma relacional de conceber o enquadramento da competição e competidores (Fligstein, 2001). Entendemos ser uma articulação teórica interessante, pois a visão sistêmica não oferece recursos para momentos de surgimento e mudança de campos de ação, mas sugere a construção de sistemas transnacionais amarrados por relações de interdependência a partir de organizações subsidiárias de corporações transnacionais (Binz \& Truffera, 2017). O que não está bem claro na teoria de campos de ação e que pode ser igualmente producente para esta teoria.

O objetivo deste artigo é analisar a influência de novas tecnologias na estrutura relacional do campo de pesquisa e desenvolvimento (P\&D), da indústria automotiva e TIC. Estamos usando construções em rede para explicar como o campo das montadoras automotivas japonesas está estruturado, tanto para dominar quanto absorver descontinuidades tecnológicas. Para atingir nosso objetivo, usamos dois espaços relacionais, o primeiro é o campo da P\&D global construído sobre as relações de cossignatários de patentes, e o segundo é o campo de narrativas tecnológicas elaborado sobre os resumos de patentes com dados de 1995 a 2016. Para isso foi necessário responder as perguntas: Há um campo da P\&D? Quais são seus principais atores? Como enquadram a ação de seus competidores? Como enquadram as novas tecnologias? Representam um verdadeiro ato "disruptivo"?

É mostrado neste artigo que montadoras constituem componentes fortemente conectados (Strong Connected Component - SCC) (Ravasz \& Barabási, 2003) como plataforma estratégica para simultaneamente competir e mitigar riscos de possíveis descontinuidades tecnológicas produzidas por inovações. O que possibilita "(a) aperfeiçoar e evoluir tecnologias existentes, (b) adquirir e desenvolver novas tecnologias e recursos e (c) integrar conhecimentos novos a existentes em produtos e soluções" (Bergek, Berggren, Magnusson, \& Hobday, 2013). Sem um campo de P\&D, com regras próprias de governança e protegido das oscilações de mercado, tal plataforma estratégica não seria possível, pois como um campo de ação de P\&D, incumbentes ou mesmo seus pares desafiantes (atores do mesmo campo) encontram certa proteção contra desafiantes externos.

Para fundamentar estes pontos, o presente estudo foi organizado como se segue: a seção 2 - Quadro Teórico foi dividida em duas subseções, a primeira apresentamos os fundamentos de um campo de ação estratégica e na segunda apresentamos constructos de campos como redes. Na seção 3 - Método apresentamos os fundamentos de análise semântica, o protocolo para a construção de componentes fortemente conectados (Strong Connected Component - SCC) e as métricas nodais adotas. 


\section{Quadro teórico}

As lentes teóricas consideradas para a interpretação dos resultados são a teoria de campo sob a perspectiva de Fligstein e McAdam (2012) e Bourdieu (2011). Ambas as teorias têm diferenças em sua construção e os pressupostos de ação são um deles. Entretanto, vemos complementaridades em certas perspectivas.

A ação concebida por Fligstein e McAdam (2012) é construída sobre a ideia de ator socialmente habilidoso. Essa categoria de ator é aquela que pode compor uma coalizão entre outros atores do campo. Desta maneira, o ator social habilidoso pode tirar vantagens simbólicas e materiais do campo. No centro desta ideia está a concepção de campo como um sistema político de coalizões (formações de grupos entre competidores), pois o ator socialmente habilidoso (incumbente ou desafiante) manterá a cooperação entre as coalizões enquanto isso Ihe conceder recompensas (Fligstein \& McAdam, 2012, p. 110). Os atores de um campo interagem na base de um senso comum sobre os propósitos do campo, como a autopreservação por exemplo. Para esses atores, a sensação de manter o campo estabilizado é a lógica que impulsiona a ação de cooperar. Portanto, competir também vem acompanhado de cooperação, principalmente se há entrada de atores externos ao campo. Nesse sentido, a formação de grupos interconectados é subproduto do campo de ação estratégica.

A ação da perspectiva de Bourdieu (2011) baseia-se no habitus, que é um conjunto individual de categorias de percepções. O ator, ao se deparar com estruturas objetivas, reage de acordo com suas categorias de percepção. O campo da perspectiva de Bourdieu (2011) é uma estrutura de poder baseada em tipos de capital e sua acumulação. De certo modo, o habitus e o campo são desconexos, a menos que as posições estruturais sejam incorporadas nas categorias de percepção do ator. A dominação de um campo está relacionada com a capacidade de alavancar mais capitais do que outros. Sendo assim, a posse de capitais é a referência para o domínio em um campo. Então, a maneira instrumental de entender a dominação no campo é entender a posse de capitais. Os capitais, segundo Bourdieu (2011), são principalmente quatro: capital econômico - refere-se à acumulação monetária; capital cultural - que se refere a um conhecimento objetivado; capital social - são as relações sociais estáveis; e capital simbólico - é uma posição estrutural que se torna uma categoria de percepção. A concepção de capitais tem uma interação circular; por exemplo, o capital cultural pode ser convertido em capital social, assim como o capital econômico pode conceder acesso ao capital cultural e social. Essa interação, no tempo, por ser uma base cognitiva, suporta a formação do capital simbólico, que é qualquer dos outros capitais incorporados como categoria de percepção (Bourdieu, 2011, p. 150).

Este conceito de estruturação de campo através da acumulação de capitais não está presente na concepção de campo de Fligstein e McAdam (2012), mas é complementar quando expressamos um campo como SCC (Variano, McCoy, \& Lipson, 2004). Nossa perspectiva com essa articulação refere-se à concepção de SCC, isto é, mesmo um componente de rede fortemente conectado, é dividido em grupos. Exatamente essa composição 
de grupos é o que confere ao SCC estabilidade (Variano, McCoy, \& Lipson, 2004). Assim, os componentes de uma rede podem representar um campo de ação estratégica. Portanto, a partir da composição da rede é possível extrair métricas nodais que diferenciam atores e permitem ao pesquisador inferir sobre seus capitais. Para Ravasz e Barabási (2003), grupos "são a consequência de uma organização hierárquica, implicando que pequenos grupos de nós se organizam de forma hierárquica em grupos cada vez maiores" com o tempo.

As formas de capitais de Bourdieu (2011) e o campo visto como um sistema político-cultural (Fligstein \& McAdam, 2012) se completam na forma de gestar interdependências. Ao mesmo tempo que a visão de Fligstein \& McAdam (2012) articula aspectos de escolha racional de uma visão política com atores habilidosos, assume características culturais que podem sustentar ou não mudanças. Os atores se entrelaçam em ações políticas e constroem narrativas para guiar essa interação (Fligstein, 1996, p. 657). Ou seja, não se trata de uma perspectiva determinística da ação, trata-se somente de aceitar que o voluntarismo ocorre nas tentativas de tomar o controle. Isso pode ter efeito ou não, mas é na habilidade social entre grupos opostos que as organizações agem interna e ambientalmente.

Outro conceito é o controle corporativo de Fligstein (2001, p.170), o qual é estabelecido por concepções de atores que visam trazer previsibilidade a relações internas e externas às organizações. Portanto, fazer com que um título de propriedade (uma patente) seja usado instrumentalmente para subsidiar a governança para além da segurança interna das organizações é um subproduto desse modelo. Através das patentes, atores habilidosos criam uma estrutura de relações externas que confere robustez e estabilidade ao campo. Um SCC é subproduto dessa concepção de controle corporativo, pois se trata de um componente no qual incumbentes e desafiantes estão em algum grau interconectados.

Neste momento é importante afirmar que em nosso entender SCCs são proxy para um campo se ele é representativo não apenas pela presença de uma relação. Temos que prestar atenção ao fato de que a relação deve representar certo tipo de relação institucional e também simbólica. Para esta pesquisa, selecionamos a ligação entre cossignatários de patentes, uma vez que este espaço de SCCs constrói o Campo Global de Pesquisa e Desenvolvimento. Nosso interesse é representar o sistema político de coalizões em sistema de inovação e, com este sistema, podemos representar as coalizões institucionais e tecnológicas, de forma a compreender que o capital cultural é a acumulação de acervo tecnológico.

\section{Tipologia da Inovação}

A fim de apoiar nossa análise de um campo de P\&D, decidimos adotar a tipologia de inovação de Shuen e Sieber (2009). Esta tipologia atravessa velhas e novas tecnologias com antigos e novos mercados. Nesta tipologia, a inovação disruptiva é uma nova tecnologia num mercado existente e antigo; Inovação incremental é tecnologia antiga em mercados antigos; a inovação radical é uma nova tecnologia em mercados novos ou emergentes; e inovação arquitetônica são tecnologias antigas em novos mercados. 
Para esta tipologia, a classificação do ambiente ou campo depende da posição dos atores. Atores automotivos estarão ao lado da estabilidade de campo e projetarão todas as barreiras para eliminar ameaças, mantendo a inovação em compasso incremental ou arquitetônico de melhoria de sua competência. Se o ator é um outsider automotivo (como atores TIC), sua atuação poderia ser a introdução de inovações disruptivas ou radicais. A principal estratégia de novos entrantes (desafiantes) é a destruição da competência do campo. No entanto, para Shuen e Sieber (2009), na melhor das hipóteses esse tipo de competição é do tipo soma zero para os incumbentes e desafiantes. Em contraposição, os autores propõem que a soma não-zero acaba por ser a escolha mais lucrativa. Com isso, a lógica de uma plataforma de inovação recombinante acaba por ser a estratégia de estabilização.

Apesar de a teoria das capacidades dinâmicas ter alguma semelhança terminológica com o campo de ação estratégica de Fligstein e McAdam (2012), ambas as teorias se referem à estratégia, mas são minimamente compatíveis no nível de análise. Então, nesta pesquisa, estamos considerando apenas a tipologia da inovação e lógica da soma não-zero para apoiar nossa interpretação dos achados.

\section{- Procedimento metodológico}

A abordagem longitudinal é importante para identificar mudanças e sua natureza no campo de ação estratégica. Adotamos duas construções temporais: (1) apresentações de métricas relacionais estruturais e nodais; e (2) grafos de relações ator-tecnologia em agrupamentos temporais. Os dados foram estruturados em painéis longitudinais de forma a identificar mudanças, atores estratégicos e coalizões tecnológicas.

Em (1) Identificamos mudanças no campo através da identificação de inflexões e alternâncias nas métricas globais da modularidade e densidade de rede. A modularidade é uma medida que varia de 0 a 100\%, onde em que 0 indica um componente tão denso que se torna impossível identificar a formação de grupos, e 100\% indica uma divisão em grupos os quais a fronteira é claramente definida pela ausência de relações entre grupos. Para identificar as organizações socialmente habilidosas, organizamos a da métrica de centralidade nodal de intermediação (betweeness) em painel temporal pelos atores mais relevantes. Em (2) organizamos os grafos de associação dos atores mais relevantes em torno das tecnologias de maior influência no campo, em que (a) é a transição da combustão interna para o elétrico - 2001-2016, e (b) a concepção do veículo autônomo após a entrada de atores TIC - 2005-2016.

Consideramos como principal fonte de dados o Derwent World Patents Index no período de 1995 a 2016. Encontramos cerca de 7211 pedidos de patentes simultaneamente registradas no Japão, China, Europa e EUA (chamadas patentes tetraédricas). A patente tetraédrica é o conjunto de patentes que tem prioridade sobre a propriedade intelectual aos seus detentores nas quatro principais regiões/países - Japão, EUA, Europa e China. O conjunto completo de patentes de 1995 a 2016 para a classe 
IPC B60 é um pouco maior que 150 mil patentes em todo o mundo. Dentre essas $150 \mathrm{~K}$ patentes, as mais citadas/relevantes somam cerca de 46K, que uma vez filtradas por ocorrências tetraédricas nos resultaram 7.211 registros que acreditamos ser a tecnologia associada à inovação. Isso porque nem todas as patentes encontram vazão na produção e por sua vez geram impacto social.

As redes foram construídas através do campo signatários em duas formas: a primeira pela co-ocorrência de signatários por patente e a segunda pela co-ocorrência de signatários por tecnologia. Esta segunda foi realizada com o suporte da ferramenta KHCoder (Higuchi, 2017), na qual analisamos os resumos de patentes e agregamos por signatários as tecnologias identificadas pela análise semântica.

Com esses dois corpora relacionais, adotamos uma forma descritiva da ação estratégica no campo, buscando identificar o espaço de competição entre atores das indústrias automotiva e TIC (Tecnologia da Inovação e Comunicações) através de suas redes.

\section{Métricas de Rede}

A análise de redes sociais (ARS), como um método, enfatiza entidades, relações e a estrutura formada. O princípio é identificar uma estrutura social como "regularidades nos padrões de relações entre entidades concretas; não é uma harmonia entre normas e valores abstratos ou uma classificação de entidades concretas por seus atributos. [Uma rede é um] agregado [dessas] regularidades de uma maneira consistente com sua natureza inerente" (White, Boorman, \& Breiger, 1976, p. 733-734).

O método identifica regularidades nos padrões através de métricas matriciais. As métricas (Hansen, Shneiderman, \& Smith, 2010) consideradas neste estudo são o número de:

(1) de entidades automotivas, que são os nós;

(2) de relações ou vínculos;

(3) de componentes conectados.

Assim, como descritores do SCC por ano, temos:

(4) o número máximo de nós do SCC;

(5) o número máximo de laços do SCC.

E dois descritores do espaço social com todos os componentes:

(6) a densidade, que é uma razão que compara o número de laços no gráfico com o número máximo de ligações que o gráfico teria se todos os nós de rede estivessem conectados uns aos outros, e

(7) modularidade, quando um grafo se divide em grupos, a modularidade é a medida de agrupamentos de um espaço social. Grafos com alta modularidade possuem conexões densas entre os vértices dentro do mesmo grupo, mas conexões esparsas entre vértices de diferentes grupos.

Com esta primeira abordagem respondemos as perguntas: (i) há um campo da P\&D? e (ii) Quais são seus principais atores? 
A última e provavelmente a métrica mais importante considerada nesta pesquisa, é a centralidade de intermediação. "O conceito de centralidade de intermediação (Betweeness) se refere a como outros atores controlam ou intermediam as relações entre pares que não estão diretamente conectados" (Knoke \& Yang, 2008, p. 67). A intermediação é a centralidade que une diferentes grupos, ou seja, a centralidade que usaremos para identificar os atores poderosos por uma estratégia relacional.

Com a abordagem de centralidade nodal respondemos as perguntas: (iii) Como seus atores enquadram a ação de seus competidores? E (iv) Como enquadram novas tecnologias?

Na Fig. 01 temos um exemplo de grafo no qual os anos de 2000 a 2005 foram agrupados. Nesse exemplo podemos ver que o SCC se subdivide em vários grupos nos quais as subsidiárias centrais são as organizações que formam os grupos e estabelecem, através de relações de parceria em patentes, coalizões com outros grupos. Os mais emblemáticos são as relações entre Toyota/Honda e Toyota/Daimler-Chrisler, tradicionais competidores no campo automotivo. É claro que a maior parte das relações ocorrem indiretamente no segundo ou terceiro grau de separação através de inventores-signatários, enquanto a menor parte é composta por relações diretas, ou seja, no primeiro grau de separação; ainda assim podemos ver que se trata de um campo dinâmico com um importante papel desempenhado por inventores-signatários. Assim, para identificar a evolução do campo, as métricas que usaremos são (4) e (5) prioritariamente.

As métricas (4) e (5) mostram a evolução do número de laços e nós do SCC, o que nos permitirá ver a evolução dinâmica do campo. Para inferir sobre suas coalizões, utilizamos o algoritmo de Clauset, Newman e Moore (2004) para simular os grupos e suas coalizões, considerando as métricas (6) e (7). A densidade, métrica (7), é altamente influenciada pelo aumento no número de nós de rede, porém, em conjunto com a modularidade, oferece maior precisão para uma análise da evolução do campo.

\section{Análise semântica}

Para analisarmos a narrativa tecnológica associada às TICs, elaboramos a análise semântica dos resumos através do KHCoder (Higuchi, 2017) das 7.211 patentes selecionadas para este estudo. Com isso, elaboramos uma análise em painel agrupando as narrativas e suas associações antes e depois do ingresso das TICs no cenário competitivo. Para tal, realizamos uma contagem de co-ocorrência de signatários por código tecnológico. Em seguida fizemos o agrupamento hierárquico (Knoke \& Yang, 2008, pp. 80-82) dos códigos de forma a identificar como as narrativas tecnológicas se associavam. Dessa forma, poderemos entender o efeito da entrada das TIC, em especial o Google, no cenário competitivo. 


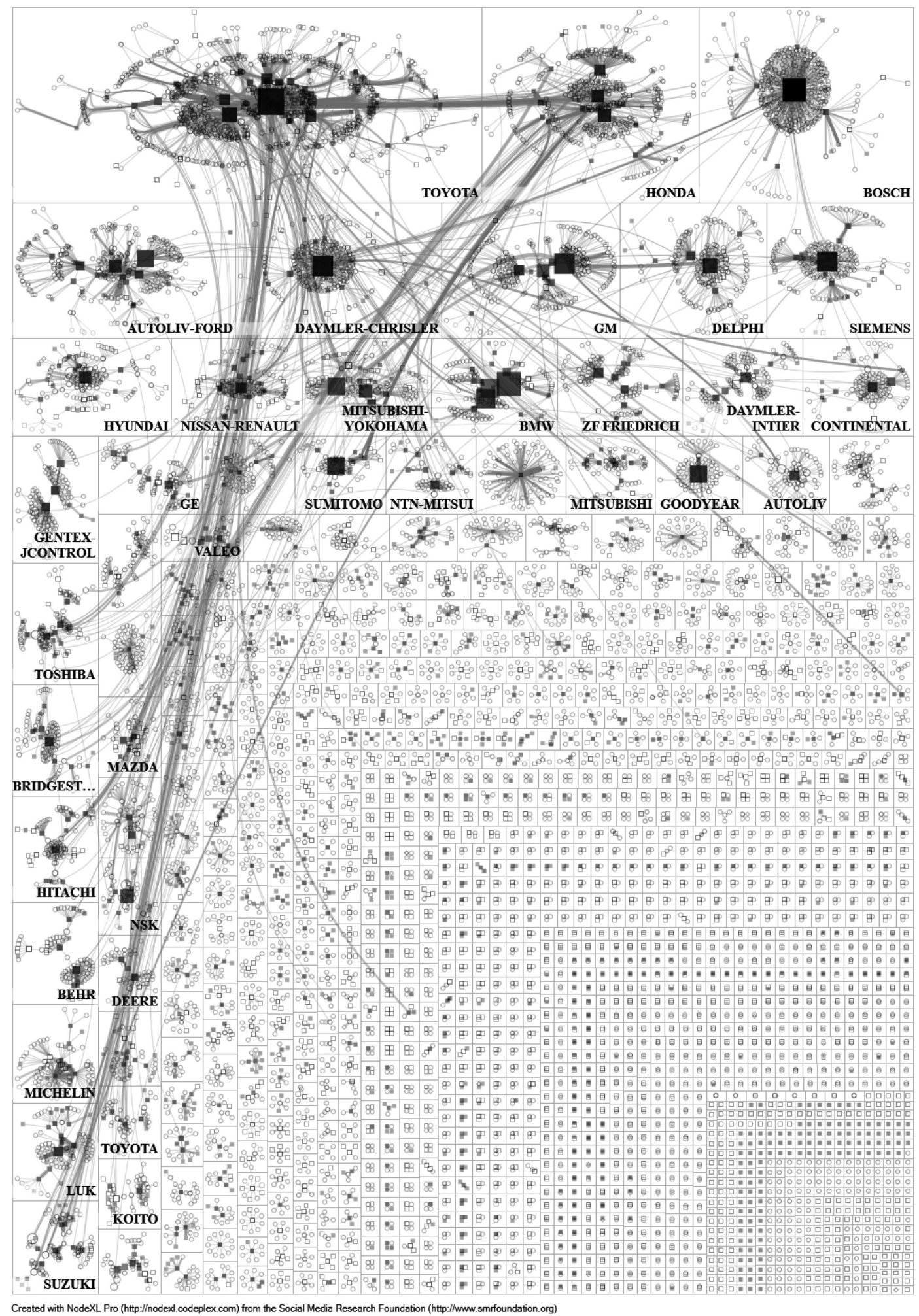

Figura 1. Grafo dos grupos formados entre montadoras entre os anos de 2000 e 2005.

Fonte: Elaborado pelos autores

Nota: Cada caixa isolada é um componente desta rede e as caixas interligadas são grupos dentre do SCC (Clauset, Newman, \& Moore, 2004). 0 nome de cada grupo foi atribuído a partir do nome da subsidiária com maior centralidade de intermediação, que também determina 0 tamanho do nó. Os nós representados por quadrados são organizações e os representados por círculos são inventores signatários de patentes. 
Os códigos foram elaborados em parte de acordo com o relatório MDOT/CAR (Hong, Wallace, \& Krueger, 2014) e em parte pelo levantamento semântico, que são:

1. Robótica - fundamental para a digitalização de operações e se refere ao elemento central do processo de digitalização da produção;

2. Veículo assistido - conceito tecnológico de automação de funções veiculares com funções limitadas de auto condução;

3. Aparatos de assistência - conceito de tecnológico associado a elaboração de acessórios para auto condução;

4. Baliza assistida - conceito específico sobra a automação da função de baliza;

5. Direção assistida - conceito associado a aspectos de automação da direção veicular;

6. Veículo autônomo - trata-se do conceito de veículo auto conduzido por sensoriamento de imagens;

7. Sensoriamento por imagens; e

8. DSCR-Veículo conectado (DSRC - Data Short Range Communication). Este último refere-se a um conceito tecnológico de gestão de tráfego centralizado. Por essa razão, seria necessária uma rede de comunicação de dados de curta distância - o DSRC.

Essa distribuição foi importante para analisar a evolução das narrativas tecnológicas por atores deste campo e elaborar a análise em painéis de tais narrativas para responder à pergunta se representam um verdadeiro ato "disruptivo".

\section{Discussão}

\section{O campo global de pesquisa e desenvolvimento}

Deve-se mencionar que o setor automotivo japonês é fortemente orientado à exportação. Com isso, suas patentes não seriam somente registradas no país de origem, mas também em qualquer país onde haja produção direta ou indireta. Desse modo, o capital sob disputa é o capital tecnológico expresso sob a forma de um título de propriedade intelectual - a patente. A patente é umas das mais antigas formas de controle corporativo, concebidas para trazer estabilidade em um ambiente competitivo (Fligstein, 2001). Isso nos mostra que as patentes, como uma concepção de controle corporativo, indicam um campo per se. Dito isto, há um forte indicativo de que a P\&D é um campo, como veremos mais adiante nos dados. Não poderemos afirmar que sempre o foi, mas podemos dizer que é no intervalo de tempo deste estudo.

Projetamos o Infográfico 1 para demonstrar as métricas sob a forma de painel cronológico do campo de P\&D japonês. Os dados estão apresentados no Infográfico 1.ii. O que queremos ressaltar aqui são as alternâncias entre as métricas estruturais da densidade e modularidade. Para isso, elaboramos 
os gráficos do Infográfico 1.i - o par, modularidade e densidade de rede. Inflexões e alternações nesse modelo indicam as mudanças no campo.

Assim, no Infográfico 1.i, temos uma formação em rede baseada nos laços de cooperação entre os signatários das patentes. No início deste projeto, esperávamos tratar os dados apenas por meio de estatísticas descritivas. De acordo com nossa expectativa, a propriedade intelectual não seria um ato cooperativo entre competidores, portanto, estávamos errados. Através da comunidade de signatários individuais e relações interorganizacionais podemos encontrar uma rede global de P\&D. O Infográfico 1.i mostra esta dinâmica.

Infográfico 1. Evolução temporal de métricas estruturais do campo da P\&D

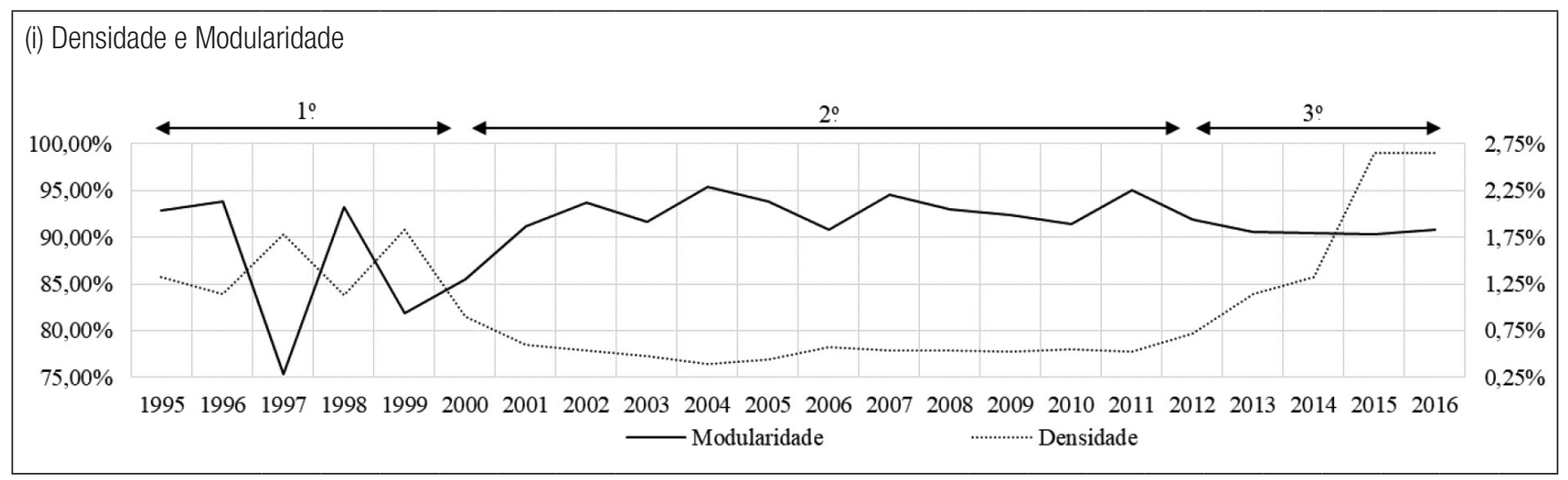

\begin{tabular}{|c|c|c|c|c|c|c|c|c|c|c|c|c|c|c|c|c|c|c|c|c|c|c|}
\hline (ii) Métricas & 1995 & 1996 & 1997 & 1998 & 1999 & 2000 & 2001 & 2002 & 2003 & 2004 & 2005 & 2006 & 2007 & \begin{tabular}{|l|}
2008 \\
\end{tabular} & 2009 & 2010 & 2011 & 2012 & 2013 & 2014 & 2015 & 2016 \\
\hline (1) Nós total & 293 & 311 & 447 & 388 & 484 & 936 & 1209 & 1333 & 1594 & 1515 & 1487 & 1381 & 1317 & 1354 & 1394 & 1335 & 1295 & 1118 & 804 & 726 & 384 & 320 \\
\hline (2) Laços total & 568 & 552 & 1782 & 848 & 2145 & 3944 & 4346 & 4731 & 6035 & 4496 & 4949 & 5425 & 4661 & 4911 & 5163 & 4915 & 4449 & 4457 & 3691 & 3479 & 1950 & 1351 \\
\hline (3) Componentes Conectados & 79 & 84 & 96 & 86 & 80 & 115 & 132 & 145 & 178 & 195 & 194 & 172 & 167 & 162 & 162 & 161 & 160 & 140 & 109 & 99 & 53 & 46 \\
\hline (4) Max Nós no SCC & 16 & 22 & 42 & 32 & 42 & 309 & 362 & 456 & 479 & 315 & 305 & 486 & 386 & 269 & 480 & 471 & 241 & 297 & 115 & 69 & 37 & 33 \\
\hline (5) Max laços no SCC & 120 & 102 & 861 & 170 & 861 & 1264 & 1363 & 1438 & 1757 & 1116 & 1040 & 3049 & 1722 & 909 & 1740 & 1652 & 933 & 1192 & 1158 & 1161 & 378 & 315 \\
\hline
\end{tabular}

Fonte: Elaborado pelos autores

Nota. (i) Densidade Estrutural e Modularidade -, (ii) Métricas gerais do gráfico.

Três momentos distintos

O campo da P\&D Global Automotiva mostra três (3) momentos distintos, e pode ser visto no Infográfico 1.i:

1. O primeiro é o período de 1995 a 2000, no qual a alternância de Q e D demonstra uma instabilidade significativa, que podemos ver através da oscilação da modularidade e densidade da rede. Isso aconteceu pela dinâmica formação e dissolução de grupos de P\&D. O que é curioso neste período é a estreita relação entre as montadoras.

2. O segundo período vai de 2001 a 2011. A Fig. 01 apresenta um extrato combinado de 2000 a 2005, o qual representa um tempo longo e estável. A principal característica observável é a ausência de alternâncias. Parece-nos um campo protegido de oscilações exógenas, especialmente quando ocorreu a depressão financeira de 2008. Ao contrário do campo de Produção, nenhuma alternância aconteceu em 2008 - que exploramos em Matui e Sacomano Neto (2017). Isso nos chamou a atenção porque nenhuma estabilidade ou 
instabilidade está desconectada do campo burocrático (Fligstein \& McAdam, 2012, p. 71). Neste período, de 2001 a 2011, mostra-se que essa estabilidade que não é possível sem uma estreita cooperação com governos. Um campo ditado apenas pelo mercado se comporta como no período de 1995 a 2000. O estado é fundamental para a estabilidade de um campo. Neste período encontramos dentre os signatários muitos inventores e as tradicionais corporações que formam a indústria automotiva. Há também institutos de pesquisa e universidades, porém são atores que pouco aparecem nos dados.

3. Finalmente, temos o terceiro período que vai de 2012 a 2016 quando encontramos uma redução estruturada da atividade de P\&D. Provavelmente se refere ao fim do ciclo dessa atividade. Entretanto, uma característica que parece ser persistente é a manutenção dos grupos de P\&D. A densidade aumenta, mas isso é resultado de uma estruturada diminuição de atividade em patentes.

Podemos dizer que houve a formação de um campo, principalmente pela composição de coalizões entre competidores. Claro que na sua maioria são relações de segundo grau, nas quais têm-se como pivô intermediários individuais - inventores listados como signatários. Trata-se de uma conexão relacional que merece uma análise dedicada. Sendo assim, algumas proposições podem ser levantadas para uma análise futura sobre o tripleto Subsidiária-Indivíduo-Subsidiária:

Proposição 01 - trata-se de uma ação de cooptação de recursos de P\&D entre competidores.

Proposição 02 - trata-se de um recurso de legitimação tecnológica na qual os ativos são concepções tecnológicas concorrentes.

\section{Ação estratégica de intermediação}

Ao longo do período desta pesquisa, um aspecto importante do campo de P\&D é descrito pelas linhas Componentes Conectados e Max Nós no SCC no Infográfico 1.ii. Vemos que existe um dinâmica anual no surgimento e desaparecimento de componentes. Isso significa que há uma grande quantidade de grupos autônomos. Isso pode ser visto no grafo da Fig. 01, em que constam todas as caixas desconectadas. Apenas para lembrar, um componente é um subgrafo isolado. Em contraste, na linha (4) Max Nós no SCC - Infográfico 1.ii, podemos ver que existe pelo menos um componente, que reúne de $20 \%$ a $30 \%$ do total de nós/laços a cada ano. Nesse sentido, temos um SCC, ou seja, um componente fortemente conectado. Mais uma vez, dentro deste componente e pelo seu porte, há a formação de grupos, nos quais a posição mais central de cada grupo é a do ator com o maior índice de intermediação (betweeness). Isso mostra que ser intermediário de capital tecnológico é uma importante ação estratégica no campo de P\&D. Sendo assim, tudo isso indica os principais atores deste campo e que sua estratégia relacional é a de acumulação de capital cultural pela coalizão.

Na Figura 2 temos os principais intermediários: os atores cuja ação está atrelada a intermediação de propriedade intelectual. Como intermediários de propriedade intelectual, tais atores também possuem o maior acervo de patentes, o que indica como esses atores convertem o capital social em 
capital cultural. Sendo assim, abre-se espaço para uma proposição sobre a concepção relacional de controle:

Proposição 03 - A conversão de capital cultural em social, e vice-versa, tem o propósito de controle sobre incertezas associadas às descontinuidades tecnológicas.

Uma vez que novas concepções tecnológicas podem surgir em qualquer lugar, a posição de intermediário confere a possibilidade de ser a escolha de afiliação de inventores independentes ou pequenas-médias organizações. Essa posição também indica os atores mais poderosos do campo.

Não estamos dizendo que os atores só precisam de capital social, mas estamos identificando que a intermediação como capital social é uma estratégia importante e se converte em capitais tecnológicos, produtivos e econômicos, conforme proposto por Bourdieu (2001). Desse modo, a governança relacional se torna uma forma de enquadrar a ação de competidores e novas tecnologias.

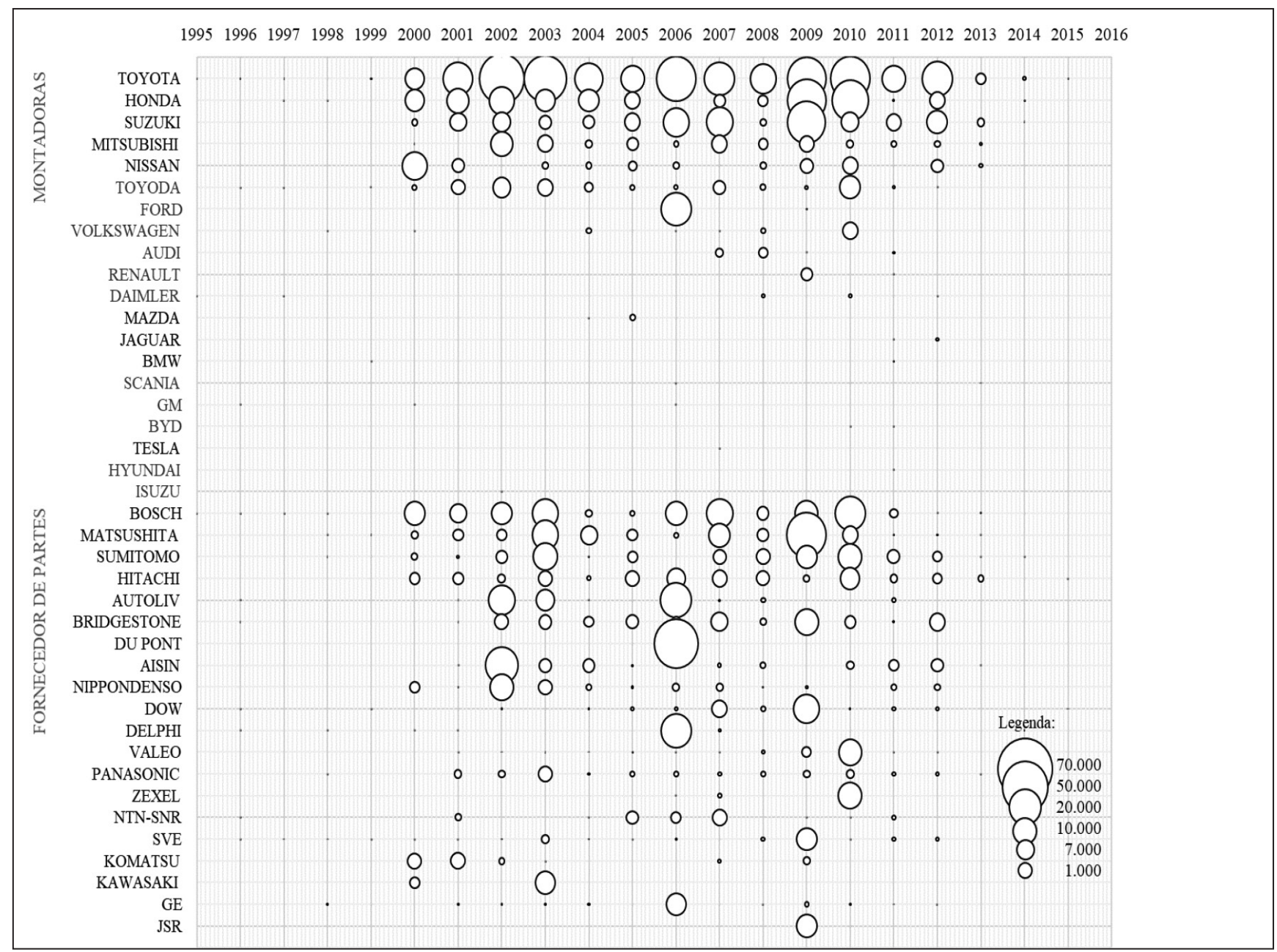

Figura 2. Centralidade de intermediação (betweeness)

Fonte: Elaborado pelos autores

Nota: As montadoras e fornecedoras de partes no campo de P\&D. Top20 para montadoras e Top20 para fornecedores de partes. 


\section{Essência global do campo de $P \& D$}

Neste ponto, é importante observar que a coleta de dados deste estudo não está restrita às montadoras japonesas. O campo de pesquisa e desenvolvimento aqui descrito é global. Quando o leitor encontrar na Figura 2 uma predominância de corporações japonesas, isso realmente significa que as organizações japonesas estão melhor posicionadas em comparação às organizações americanas, europeias e chinesas. Isso mostra uma distinção clara entre tais montadoras: o que as diferencia é a estratégia relacional baseada na intermediação (betweeness). Isso trata-se de um achado que não era esperado nesta pesquisa.

O achado final, mas não menos importante, é a quase ausência de atores da Tecnologia da Informação e Comunicação (TIC) como atores intermediários no campo da P\&D. Aparentemente, o setor de TIC se desenvolve marginalmente no campo da P\&D automotiva. O que era esperado, devido ao fato de ser um campo tecnológico centenário, em que os atores deste campo se dividem em várias linhas tecnológicas.

Portanto, pode-se dizer que as TICs enfatizam apenas alguns aspectos tecnológicos importantes para as mudanças no campo e representam uma fração do total de tecnologias necessárias para a concepção de um veículo.

\subsection{As TICs no campo de $P \& D$}

Da perspectiva da tecnologia de condução autônoma, os atores TIC são os novos entrantes no campo automotivo. Como recém-chegados, tais atores não são atores centrais no setor automotivo. Assim, as TICs não contam com uma plataforma automotiva sobre a qual se constroem inovações. Por outro lado, o capital simbólico dos atores de TIC vem do campo de ação da internet. Nesse campo, atores de TIC como o Google, a Apple e a Microsoft são vistos como atores poderosos, o que pode levar o senso comum a interpretá-los como atores poderosos em qualquer outro campo de ação. No entanto, o que podemos ver nesta análise, especialmente no campo de P\&D, é que esses atores desempenham um papel marginal. Um aspecto já destacado na seção anterior é a ausência absoluta de TICs como intermediários, pelo menos até 2016. No entanto, eles são signatários de patentes sob o código IPC B60 - automotivo.

$\mathrm{Na}$ Figura 03.a temos os principais atores do campo e suas associações aos conceitos tecnológicos adotados neste estudo. Vemos que o Google somente aparece como signatário de patentes tetraédricas a partir de 2009. Antes disso se tratava de um campo no qual a narrativa tecnológica era dirigida por atores tradicionais do setor automotivo. $\mathrm{Na}$ Figura 3.b do período de 2005 a 2008, vemos que a narrativa tecnológica era então dominada pelo conceito do veículo assistido. O veículo assistido se refere à autonomia limitada a algumas funções de dirigibilidade. Nesse período os principais intermediários tecnológicos foram Toyota, Nissan e Bosch. No período seguinte, de 2009 a 2012, o campo permanece fortemente influenciado pelo conceito tecnológico do veículo assistido, porém uma nova narrativa tecnológica emerge, nucleada pelos conceitos 
tecnológicos robótica, veículo autônomo e sensoriamento por imagens. Neste período, além dos tradicionais intermediários, temos o surgimento do Google, GM e Daimler como intermediários do conceito tecnológico de veículo autônomo. No período que vai de 2013 a 2016 ocorre um certo esvaziamento de atores tradicionais do setor automotivo. Esta redução de atividades ocorre em todas as narrativas tecnológicas do campo, não apenas nas analisadas aqui.

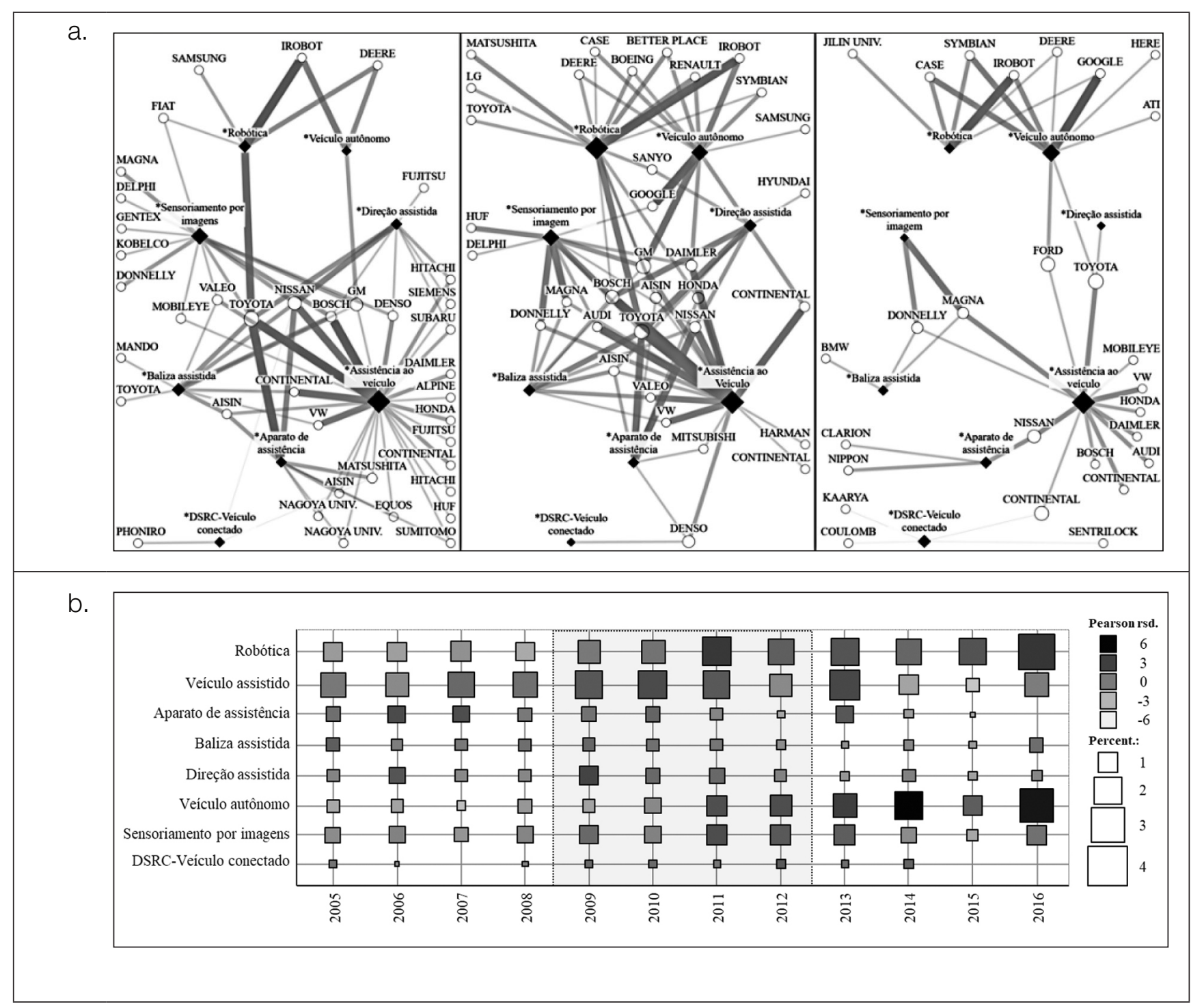

Figura 3. Conceitos tecnológicos, a) redes de afiliações e b) influência no tempo Fonte: Elaborado pelos autores

Nota: a) rede de co-ocorrência conceitos tecnológicos organizações e b) tabulação cruzada número de patentes por conceito tecnológico.

Entretanto, fica evidente que os atores do campo se dividem entre as concepções tecnológicas e as narrativas contraditórias começam a indicar novas polarizações.

Para analisar como o campo reage às novas tecnologias vamos nos reportar à Figura 04. Na Figura04.a tem-se como as narrativas se associavam 
no período de 2005 a2008. Aqui vemos dois grupos hierárquicos subordinados ao conceito tecnológico veículo assistido, no qual o primeiro grupo indica que o conceito tecnológico veículo autônomo estava subordinado ao conceito veículo conectado. Essa é uma ideia dos anos 1990, em que a autonomia seria gestada por um serviço centralizado, como numa ferrovia (Hong, Wallace, \& Krueger, 2014).

Já a narrativa no período de 2009 a 2012 se agrupa de forma diferente neste período. O conceito de veículo assistido passa a ser associado aos fundamentos tecnológicos da robótica e permanece hierarquicamente mais amplo, inclusive sobre o conceito de veículo autônomo que está associado ao sensoriamento por imagens. Assim, esse é exatamente o campo por onde o Google decide fundamentar sua tecnologia.

A narrativa do período que vai de 2013 a 2016 pode referir-se ao fim de um ciclo de P\&D. Trata-se de uma fragilidade deste estudo, pois não temos informações sobre o que motivou esse esvaziamento do campo.

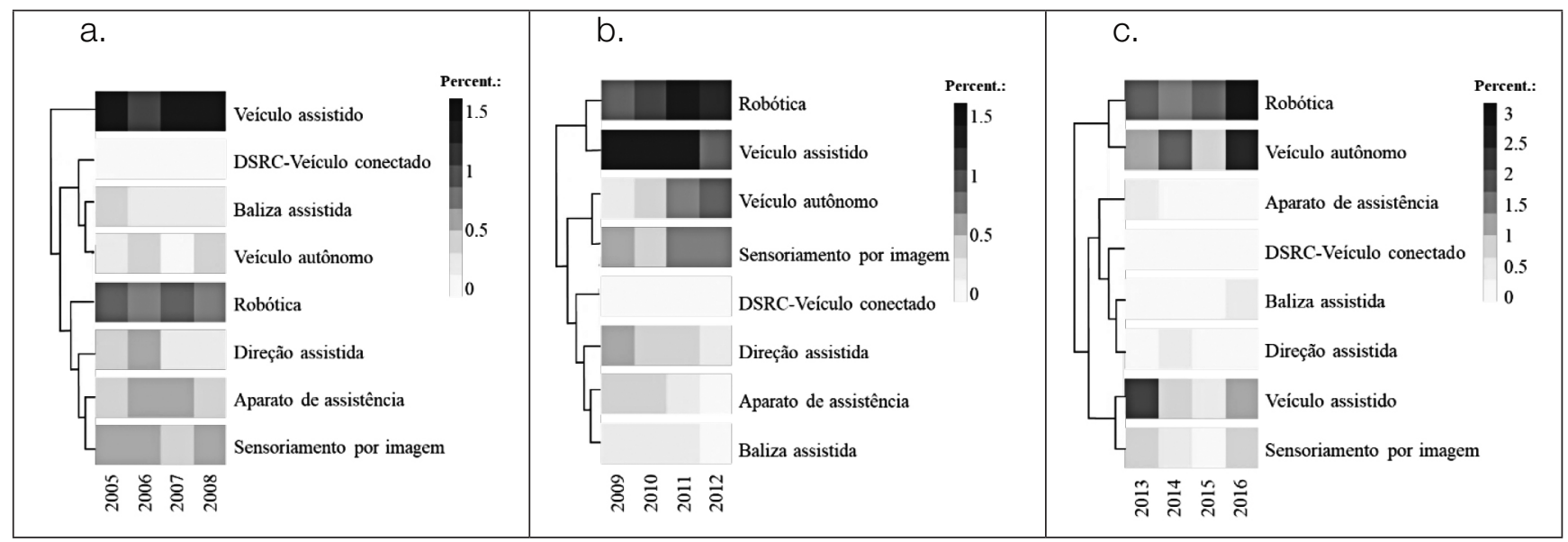

Figura 4. Narrativas tecnológicas antes e após a entrada de TICs ao cenário automotivo.

Fonte: Elaborado pelos autores.

Nota: Patentes recuperadas por período: a) 132 documentos em 2737; b) 172 documentos em 2663; e c) 80 documentos em 1359

Como mencionamos no início deste trabalho, estamos falando de um campo de ação. Assim, a ação de um ator pode influenciar o campo, mas não determina seus rumos. O enquadramento de narrativas mostra que atores desafiantes podem oportunamente adotar concepções tecnológicas antagônicas. Como mostra o avanço da GM pelo conceito de veículo autônomo semelhante ao do Google, e contra a concepção tecnológica do veículo assistido que é defendido pela Toyota. Um SCC é sobretudo uma concepção de controle sobre descontinuidades tecnológicas. Quando atores poderosos adotam novas concepções tecnológicas, o efeito é o de atrair outros atores poderosos, e com isso abduzir inovações de forma a incorporá-las ao sistema. A hipótese de "(a) aperfeiçoar e evoluir tecnologias existentes, (b) adquirir e desenvolver novas tecnologias e recursos e (c) integrar conhecimentos novos a existentes em produtos e soluções" (Bergek, Berggren, Magnusson, \& Hobday, 2013) em um campo de ação acaba por se realizar. 
Com certeza, a P\&D necessária para se ter um carro vai além da P\&D necessária para desenvolver conceitos tecnológicos que o tornarão autônomo. Isso fica claro na proporção de patentes correspondentes a concepções tecnológicas consideradas neste estudo - cerca de 6\% de 7.211 patentes. Ao mesmo tempo, para transformar o carro em uma plataforma conectada a um ecossistema da internet, os TICs têm diferentes esforços de P\&D, quando comparados ao P\&D das montadoras tradicionais.

Com isso, aparentemente estamos diante de um processo de inovação arquitetônica para os dois setores, automotivo e TIC. Isso porque o carro virou um objeto de competências recombinadas. Na verdade, parece que as TICs e as montadoras de veículos estão criando um ponto de integração de competências. Portanto, não se trata de uma inovação disruptiva, ou mesmo radical de nenhuma das perspectivas - TICs e montadoras de veículos, é uma inovação arquitetônica.

\section{Considerações finais}

Nosso objetivo foi analisar a influência de novas tecnologias na estrutura relacional do campo automotivo de P\&D. As estruturas relacionais analisadas nesta pesquisa nos mostraram que a formação de grupos hierárquicos em CCS evidencia a ação estratégica das montadoras em estabilizar o campo de P\&D (Ravasz \& Barabási, 2003). Em ambos os campos, podemos ver diferentes evidências de estabilização. No campo da Produção, a estabilização é mostrada pela linha de tendência logarítmica. Temos que lembrar que o campo de produção é predominantemente influenciado pelas incertezas do mercado. Ademais, campo de pesquisa e desenvolvimento mostra estabilidade por mais de dez anos. Talvez esta seja uma conexão com o governo japonês. Não podemos ter certeza, pois essa é uma das nossas limitações. Nós não temos relações que conectam o campo da P\&D ao governo japonês. O que temos é um pedaço da década de 1990, na qual os fabricantes de automóveis são os principais responsáveis pelas patentes. Este período foi marcado pela alta instabilidade no campo de P\&D, sendo assim, em nossa linha de pesquisa, avaliamos o que torna o campo de P\&D estável na década de 2000.

A intermediação (Betweeness) dos grupos é uma importante ação estratégica no setor automotivo japonês. Não apenas no campo de produção, mas também no campo da P\&D. Além disso, a intermediação como capital social é uma estratégia que interage com o capital tecnológico - o capital cultural segundo Bourdieu (2011). Essa evidência empírica corrobora as formas de capital e sua troca circular.

Outro achado importante nesta pesquisa é uma clara distinção entre as montadoras japonesas e as demais montadoras - americanas, europeias e chinesas - a estratégia relacional baseada na intermediação (betweeness) as distingue. Parece que a cooperação não está na taxa de concorrência para as montadoras americanas, europeias e chinesas.

Além da natureza intermediária dos atores, outro achado deste estudo está relacionado às relações recíprocas. A relação recíproca está relacionada 
principalmente aos negócios amarrados entre os fabricantes de automóveis mais fortes. Isso demonstra como as montadoras japonesas reorganizaram uma nova configuração de robustez e estabilidade no campo. Eles fizeram isso através da ligação entre negócios - relações recíprocas. Aparentemente, os atores da tecnologia de informação e comunicação (TIC) são jogadores periféricos no setor automobilístico.

Finalmente, se olharmos para a indústria automotiva como um todo, trata-se de uma "inovação arquitetônica, [aonde] tecnologias antigas em novos mercados, recombinadas e reempacotando tecnologias existentes dentro de arquiteturas redesenhadas de produtos e sistemas para alcançar novos segmentos e nichos de mercado" (Shuen \& Sieber, 2009, p. 131), cujo fim pode ser uma plataforma de inovação em que "as empresas inovaram fornecendo plataformas de onde inovações geradas externamente podem resultar, e onde usuários e ecossistemas afiliados, terceiros desenvolvedores, e prestadores de serviços podem formar comunidades inovadoras" (Shuen \& Sieber, 2009, p. 140).

\section{Agradecimentos}

Agradecemos à FAPESP e ao CNPq pelo suporte financeiro a divulgação internacional deste trabalho. Agradecemos também ao editor e equipe de pareceristas da RECADM que contribuíram com sugestões e críticas, assim como o empenho de pareceristas do SEMEAD 2017 que, de forma generosa, indicaram o artigo para o sistema de Fast Track.

\section{Referências}

Bail, C. A. (2014, julho). The cultural environment: measuring culture with big data. Theory and Society, 43(3-4), 465-482. doi:https://doi-org.ez31.periodicos.capes. gov.br/10.1007/s11186-014-9216-5

Bergek, A., Berggren, C., Magnusson, T., \& Hobday, M. (2013). Technological discontinuities and the challenge for incumbent firms: Destruction, disruption or creative accumulation? Research Policy, 42, 1210-1224. doi:http://dx.doi. org/10.1016/j.respol.2013.02.009

Binz, C., \& Truffera, B. (2017). Global Innovation Systems - A conceptual framework for innovation dynamics in transnational contexts. Research Policy, 46, 1284-1298. doi:http://dx.doi.org/10.1016/j.respol.2017.05.012

Bourdieu, P. (2001). The forms of capital. In J. Richardson, M. Granovetter, \& R. Swedberg (Eds.), The Sociology of Economic Life (2nd ed.), Cambridge: Westview Press.

Clauset, A., Newman, M. E., \& Moore, C. (2004, dezembro). Finding community structure in very large networks. Physical Review E, 70(6), 1-6.

Dijk, M., PeterWells, \& Kemp, R. (2016). Will the momentum of the electric car last? Testing an hypothesis on disruptive innovation. Technological Forecasting \& Social Change, 105, 77-88. doi:http://dx.doi.org/10.1016/j.techfore.2016.01.013 
Fellows, R., \& Liu, A. M. (2016, September). 'What does this mean'? Sensemaking in the strategic action field of construction. Construction Management and Economics, 35(8-9), 578-596. doi:10.1080/01446193.2016.1231409

Fligstein, N. (1996, August). Markets As Politics: A Political-Cultural Approach To Market Institutions. American Sociological Review, 61(4), 656-673.

Fligstein, N. (2001). The architecture of markets (1st ed.). New Jersey: Princeton University Press.

Fligstein, N., \& McAdam, D. (2012). A Theory of Fields (1st Edition ed.). New York: Oxford University Press.

Franssen, T., \& Kuipers, G. (2013, February). Coping with uncertainty, abundance and strife: Decision-making processes of Dutch acquisition editors in the global market for translations. Poetics, 41(1), 48-74. doi:https://doi.org/10.1016/j.poetic.2012.11.001

Garlaschelli, D., \& Loffredo, M. I. (2004). Patterns of link reciprocity in directed networks. Physical review letters, 93(26), 268701.

Hansen, D. L., Shneiderman, B., \& Smith, M. A. (2010). Analyzing Social Media Networks with NodeXL (1st. ed.). Burlington: Morgan Kaufmann.

Higuchi, K. (2017). KH Coder 3 Reference Manual. Ritsumeikan University.

Hölzl, W., \& Janger, J. (2014). Distance to the frontier and the perception of innovation barriers across European countries. Research Policy, 43, 707-725. doi:http://dx.doi. org/10.1016/j.respol.2013.10.001

Hong, Q., Wallace, R., \& Krueger, G. (2014). Connected vs. Automated Vehicles as Generators of Useful Data (Relatório de Pesquisa/2014), Michigan, MI, Michigan Department of Transportation, MDOT, Center for Automotive Research, CAR..

Knoke, D., \& Yang, S. (2008). Social Network Analysis (2nd. ed.). Thousand Oaks: SAGE.

Lazzarini, S. (2011). Capitalismo de laços (1a. ed.). Ro de Janeiro: Campus.

Lee, J.-Y., Bachrach, D. G., \& Lewis, K. (2014, May-Jun). Social Network Ties, Transactive Memory, and Performance in Groups. Organization Science, 25(3), 951 967. doi:10.1287/orsc.2013.0884

Lundvall, B. $\AA$ A. (2007). National Innovation Systems - Analytical Concept and Development Tool. Industry and Innovation, 14(1), 95-119. doi: 10.1080/ 13662710601130863

Marti, G. (2017, March). New Concepts for New Dynamics: Generating Theory for the Study of Religious Innovation and Social Change. Journal for the Scientific Study of Religion, 56(1), 6-18. doi:10.1111/jssr.12325

Matui, P. C., \& Sacomano Neto, M. (2017). Relational governance of Japanese automotive industry: modularity and multiregional strategic action field. IJATM, 17(4), 430-451. doi:10.1504/IJATM.2017.10010247

Mitnick, B. M., \& Ryan, R. C. (Apr de 2015). On making meanings: Curators, social assembly, and mashups. Strategic Organization, 13(2), 141-152. doi:https://doi-org. ez31.periodicos.capes.gov.br/10.1177/1476127015580310

Newman, M. E. (2006). Modularity and community structure in networks. PNAS, 103(23), 8577-8582. 
Ravasz, E., \& Barabási, A.-L. (2003). Hierarchical organization in complex networks. Physical Review, 67(2), 026112.

Schuelke-Leech, B.-A. (2018). A model for understanding the orders of magnitude of disruptive technologies. Technological Forecasting \& Social Change, 129, 261-274. doi:https://doi.org/10.1016/j.techfore.2017.09.033

Shuen, A., \& Sieber, S. (2009). Orchestrating the New Dynamic Capabilities. IESE Insights, Fourth Quarter (3), 58-65.

Teece, D. J., Pisano, G., \& Shuen, A. (1997). Dynamic Capabilities and Strategic Management. Strategic Management Journal, 18(7), 509-533.

Variano, E. A., McCoy, J. H., \& Lipson, H. (2004, May 7). Networks, Dynamics, and Modularity. Physical Review Letters, 92(18), 1-4.

White, H. C., Boorman, S. A., \& Breiger, R. L. (1976). Social Structure from Multiple Networks. I. Blockmodels of Roles and Positions. American Journal of Sociology, 81(4), 730-780. 